\title{
Complexação de íons de metais por matéria orgânica dissolvida: modelagem e aplicação em sistemas reais
}

\author{
Paulo Sérgio Silva BEZERRA르, Luís Roberto TAKIYAMA², Cícero Wellington Brito BEZERRA ${ }^{3}$
}

\begin{abstract}
RESUMO
Os íons de metais pesados são conhecidos tanto pela sua importância fisiológica e industrial, bem como pelo risco ambiental e à saúde humana. Para elucidar o comportamento dessas espécies nos corpos hídricos, os quais recebem grande parte da descarga de metais, seja de origem antrópica ou por fontes naturais, é necessário entender as interações que elas apresentam com o meio, principalmente a especiação química. Um dos mais importantes processos pelos quais passam as espécies metálicas em corpos aquáticos naturais é a interação com a matéria orgânica dissolvida (MOD), que pode ser por adsorção, reações de troca iônica ou por complexação. Neste trabalho foram realizados vários experimentos com o objetivo de descrever o comportamento da complexação de três importantes cátions $\mathrm{Cu}(\mathrm{II}), \mathrm{Cd}(\mathrm{II})$ e $\mathrm{Pb}(\mathrm{II})$ com a matéria orgânica (ácido húmico comercial), sob condiçôes diversas de força iônica em meio tamponado. Os resultados foram avaliados de acordo com o modelo de van den Berg e Kramer para a complexação de metais. O modelo foi aplicado na determinação da capacidade de complexação dos íons em amostras reais, oriundas de três rios maranhenses que integram a Amazônia legal: Itapecuru, Bacanga e Pericumã. Nas águas dos rios utilizou-se o parâmetro carbono orgânico dissolvido (COD) para expressar a MOD. Os resultados confirmaram forte interação entre a MOD e íons de metais pesados e que o modelo de van den Berg e Kramer é satisfatório para se estimar a constante de complexação $(\mathrm{K})$ e a concentração de sítios de complexação $(\mathrm{Lt})$. Nas amostras simuladas em laboratório a ordem de complexação dos metais foi $\mathrm{Cu}(\mathrm{II})>\mathrm{Cd}(\mathrm{II})>\mathrm{Pb}(\mathrm{II})$ e a capacidade de complexação mostrou ser linear em função da concentração de ácido húmico comercial. Acredita-se que por ter menor raio iônico, o íon $\mathrm{Cu}$ (II) possui maior afinidade com os sítios de complexação. Nas amostras retiradas dos corpos aquáticos, observou-se que o rio com maior concentração de COD (rio Bacanga) apresentou maior capacidade de complexação; entretanto, a ordem foi $\mathrm{Pb}(\mathrm{II})>\mathrm{Cu}(\mathrm{II})>\mathrm{Cd}(\mathrm{II})$ provavelmente devido à presença de íons $\mathrm{Cu}(\mathrm{II})$ em maior quantidade nas águas dos rios.
\end{abstract}

PALAVRAS-CHAVE: Matéria orgânica dissolvida, Íons de metais, Ácidos húmicos, Equilíbrio de complexação

\section{Complexation of metal ions by dissolved organic matter: modeling and application to real systems}

\begin{abstract}
Metal ions are known by their physiological and industrial importance as well as by their environmental risk aspects. To elucidate the behavior of these elements in superficial water bodies, it is necessary to understand the various occurring interactions, mainly those related to the chemical speciation. One of the most important processes that metal species undergo in natural aquatic bodies is their interaction with dissolved organic matter (DOM), which can happen through adsorption, ionic exchange and complexation. In this work, laboratory experiments with $\mathrm{Cu}(\mathrm{II}), \mathrm{Pb}(\mathrm{II})$ and $\mathrm{Cd}(\mathrm{II})$ ions and commercial humic acid were made under various conditions (ionic strength and buffering) to test the van den Berg and Kramer complexation model. After the simulation, the model was used to determine the $\mathrm{Cu}(\mathrm{II}), \mathrm{Pb}(\mathrm{II})$ and $\mathrm{Cd}(\mathrm{II})$ complexation capacity of natural waters from three important rivers in the state of Maranhao, Brazil (Itapecuru, Bacanga and Pericuma rivers). The dissolved organic carbon (DOC) concentration was used to express the DOM concentration. The results confirmed that there is a strong interaction between the DOM and metal ions, and that the van den Berg and Kramer model (with one coordination site) is suitable for estimating the complexation constant $(\mathrm{K})$ and the ligand site concentration $(\mathrm{L})$. The simulated water samples used in the laboratory showed the complexation order: $\mathrm{Cu}(\mathrm{II})>\mathrm{Cd}(\mathrm{II})>\mathrm{Pb}(\mathrm{II})$; and the complexation capacity was directly proportional to the humic acid concentration. We believe that the smaller $\mathrm{Cu}(\mathrm{II})$ ionic radius yields a stronger affinity with the DOM. In the experiments with natural waters, the river with more DOC (Bacanga river) showed better complexation capacity; however, the order was $\mathrm{Pb}(\mathrm{II})>$ $\mathrm{Cu}(\mathrm{II})>\mathrm{Cd}$ (II) probably because of the presence of appreciable concentrations of $\mathrm{Cu}(\mathrm{II})$ in natural waters.
\end{abstract}

KEY WORDSDissolved organic matter, Metal ions, humic acids, Complexation equilibrium

\footnotetext{
1 Universidade Federal do Maranhão, Centro de Ciências Agrárias e Ambientais. Campus de Chapadinha, BR. 222 - KM 04, s/n, 65500-000 - Chapadinha, Maranhão - Brasil. E-mail: paulossbezerra@hotmail.com

2 Instituto de Pesquisas Cientificas e Tecnológicas do Estado do Amapá - IEPA. Rd. J.K. km 10, Fazendinha, 68900-005 - Macapá - AP. E-mail: luis.takiyama@iepa.ap.gov.br

${ }^{3}$ Universidade Federal do Maranhão, Departamento de Química. Av. dos Portugueses, S/N, Campus do Bacanga, 65080-040, São Luís, Maranhão - Brasil. E-mail: cicero@ufma.br
} 


\section{INTRODUÇÃO}

A ocorrência acentuada de íons de metais em ambientes aquáticos deve-se, principalmente, às atividades industriais (Abdel, 2007; Shazili, 2006). A contaminação de ambientes por estes metais representa um risco não apenas para a população humana, mas também para a sobrevivência de outros animais, microorganismos e plantas. $\mathrm{O}$ conhecimento e controle da descarga desses poluentes são, portanto, um fator importante para a proteção do meio ambiente (Martiniano, 2008; Abdel, 2007; Shazili, 2006).

Entretanto, a certeza de que níveis de ocorrência destes cátions não representam riscos à vida exige, antes de tudo, o entendimento dos seus comportamentos, nos diversos ecossistemas. Devido às peculiaridades e complexidade de cada corpo aquático, ainda não foi possível a elucidação completa da influência da complexação na especiação (Cances, 2003; Allen \& Hansen, 1996) de íons de metais. Deste modo, estudos individuais são necessários para a elucidação das reações de complexação que ocorrem em um determinado ambiente. Dentre estes estudos, destaca-se o da interação da matéria orgânica, Substâncias Húmicas (SH) e Fúlvicas (SF), com os íons de metais presentes no meio, a qual tem um efeito marcante sobre a especiação e toxicidade de íons de metais para a biota (Sánchez-Marín, 2007; Voelker, 2001; Stum, 1992; Stumm \& Morgan, 1996). A interação M-SH é influenciada por variáveis, tais como: carga e heterogeneidade química do material húmico. Como conseqüência, a interação dos íons metálicos com este material não depende somente da concentração das citadas espécies, mas também da força iônica, podendo ser descrito empiricamente pela seletividade da função ligante (Sánchez-Marín, 2007; Nederiof, 1993; Stum, 1992; Stumm \& Morgan, 1996). As interaçōes podem acontecer através de adsorção, reaçôes de troca catiônica e por complexação. As reaçōes de complexação são as mais importantes, pois afetam profundamente a geoquímica dos íons metálicos, modificando a sua solubilidade, carga e potencial redox. Essas mudanças influenciam a biodisponibilidade, transporte e migração dos metais nos ecossistemas aquáticos.

Um método desenvolvido por van den Berg e Kramer (1979) consiste em representar a relação $M_{\text {live }}$ vs $M_{\text {livrel }}$ l $\mathrm{M}_{\text {complexado }}$ para complexos de estequiometria 1:1. Para processos envolvendo apenas um sítio de coordenação, é obtida uma função linear, onde a constante aparente de formação (K') do complexo é a razão entre o coeficiente angular e linear e a quantidade de ligante total (Lt), é o inverso do coeficiente angular (Eq. 01).

$$
\mathrm{M} / \mathrm{ML}=1 /\left(\mathrm{K}^{\prime} \mathrm{Lt}\right)+\mathrm{M} / \mathrm{Lt}
$$

Onde: $\mathrm{M}=$ concentração do metal livre

$\mathrm{ML}=$ concentração do metal complexado
$\mathrm{K}^{\prime}=$ constante de estabilidade condicional

$\mathrm{Lt}=$ capacidade de complexação

A capacidade de complexação do sistema também pode ser resultado da formação de uma série de complexos 1:1 com ligantes e constantes de estabilidades diferentes. Um novo quadro fornece quatro parâmetro experimentalmente disponíveis, duas interseções e duas inclinaçōes, são elas $\left(\mathrm{L}_{1}\right.$, $\mathrm{L}_{2}, \mathrm{~K}_{1}$ e $\mathrm{K}_{2}$ ) onde $\mathrm{L}_{1}$ e $\mathrm{L}_{2}$ são quantidades dos ligantes e $\mathrm{K}_{1} \mathrm{e}$ $\mathrm{K}_{2}$ são constantes de formação condicionais para cada sítio. A capacidade de complexação total do ligante é o somatório das capacidades de complexação $\left(\mathrm{L}_{\mathrm{t}}=\mathrm{L}_{1}+\mathrm{L}_{2}\right)$ para cada sítio (Ellwood e van den Berg, 2000; van den Berg e Kramer, 1979).

Neste trabalho, curvas $M_{\text {livre }}$ vs $M_{\text {livre }} / M_{\text {complexado }}$, foram construídas para a determinação da constante de formação dos complexos (M-MOD), e para a determinação da quantidade total de ligante na forma complexada nas amostras analisadas (Ellwood e van den Berg, 2000; Westall, 1995). O comportamento dos íons divalentes de cobre, cádmio e chumbo foi estudado, em presença da matéria orgânica dissolvida (MOD). Devido à complexidade das substâncias húmicas $(\mathrm{SH})$ e, apesar de vários esforços e diversas metodologias já terem sido utilizadas para este fim, a elucidação da natureza das interaçôes com íons metálicos ainda é assunto atual e de reconhecida importância.

A contribuição do presente trabalho foi de determinar a capacidade da matéria orgânica dissolvida em complexar os íons metálicos $\mathrm{Cu}(\mathrm{II}), \mathrm{Cd}(\mathrm{II})$ e $\mathrm{Pb}(\mathrm{II})$ em amostras de águas superficiais de três importante rios maranhenses, que integram a amazônia legal: Itapecuru, Bacanga e Pericumã, com o objetivo de estabelecer o quanto tais ambientes suportariam de carga destes íons. Adicionalmente, este estudo também pode auxiliar na modelagem e elaboração de estratégias para o controle destes metais em outros ambientes aquáticos (Santana, 2007; Ruzic, 1980).

\section{PARTE EXPERIMENTAL}

Todos os reagentes empregados foram de pureza analítica e procedência ALDRICH, MERCK ou SIGMA. A água utilizada no preparo das soluções foi purificada num ultrapurificador do sistema NANOPURE (Ultrapure Water Sistema), modelo D4741.

As soluçôes padrões dos íons metálicos $\left(1,0 \times 10^{-2} \mathrm{~mol}\right.$ $\left.\mathrm{L}^{-1}\right) \mathrm{Cu}(\mathrm{II}), \mathrm{Cd}(\mathrm{II})$ e $\mathrm{Pb}(\mathrm{II})$, foram preparadas a partir dos seguintes sais: Sulfato de Cobre pentahidratado, $\mathrm{Cu}\left(\mathrm{SO}_{4}\right)_{2} \cdot 5 \mathrm{H}_{2} \mathrm{O}$ (MERCK), Nitrato de Cádmio tetrahidratado - $\mathrm{Cd}\left(\mathrm{NO}_{3}\right)_{2} \cdot 4 \mathrm{H}_{2} \mathrm{O}$ (MERCK), Nitrato de Chumbo, $\mathrm{Pb}\left(\mathrm{NO}_{3}\right)_{2}$ (MERCK), todas acidificadas com ácido nítrico (SIGMA) a $1,5 \%(\mathrm{v} / \mathrm{v})$.

Os ácidos húmicos utilizados como padrão foram do tipo comercial (ALDRICH). Para a manutenção do pH 6,0 
utilizou-se nas soluções o tampão PIPES - piperazina N-N' ácido 2 -etanosulfônico, sal dissódico (SIGMA, pKa = 6,80) na concentração de $7,5 \times 10^{-5} \mathrm{~mol} \mathrm{~L}^{-1}$.

A técnica utilizada para a determinação das capacidades de complexação e constantes de estabilidade aparente foi a titulação potenciométrica, utilizando eletrodos de íons seletivos. As titulaçôes e as medidas do $\mathrm{pH}$ das soluções de trabalho foram realizadas em um Potenciômetro Micronal, Modelo B 47, utilizando-se um eletrodo $\mathrm{Ag} / \mathrm{AgCl}$ de dupla junção, modelo Orion 90-02, como eletrodo de referência, e para cada experimento utilizava-se um eletrodo de íon-seletivo do próprio íon estudado, modelo Orion 94-92. Um eletrodo de vidro combinado marca Orion, modelo Perphect 9242, foi empregado para o estabelecimento e controle do $\mathrm{pH}$ das soluçôes. Microburetas graduadas (resolução de 0,01, com volume total de 2,00 mL), Socorex, foram utilizadas para o preparo de soluções. As titulações foram realizadas em soluçôes sob agitação constante, para a qual se empregou um agitador magnético. Todos os experimentos foram realizados em triplicada e à temperatura de $25 \pm 1{ }^{\circ} \mathrm{C}$.

Os frascos utilizados nas coletas das amostras foram de polietileno, os quais foram devidamente limpos e deixados em contato com ácido nítrico $10 \%$, durante 24 horas. Todas as amostras foram coletadas no período de estiagem e entre os meses de Agosto e Dezembro. Para mais cobertura do trabalho e análise dos resultados, três importantes rios foram pesquisados: Itapecuru, Bacanga e Pericumã. A temperatura das amostras foi medida "in situ". Todas as amostras, depois de filtradas, foram mantidas sob refrigeração, com temperatura em torno de $-4^{\circ} \mathrm{C}$.

Parâmetros físico-químicos investigados: em todas as amostras foram realizadas determinações de: $\mathrm{pH}$, condutividade, íons sulfato, cloreto, sólidos totais, sólidos em suspensão, $\mathrm{CO}_{2}$ livre e COD (Carbono Orgânico Dissolvido). Os ensaios seguiram as metodologias propostas pela literatura (Arcos, 1998) e estão apresentados na Tabela 1.

Tabela 1 -Características físico-químicas das amostras de água coletadas nos rios Pericumã, Bacanga e Itapecuru.

\begin{tabular}{lccc}
\hline Parâmetros & Rio Pericumã & Rio Bacanga & Rio Itapecuru \\
\hline $\mathrm{pH}$ & 4,15 & 8,64 & 7,06 \\
Condutividade $\left(\mu \mathrm{S} . \mathrm{cm}^{-1}\right)$ & 400,0 & 2800 & 72,20 \\
$\mathrm{SO}_{4}{ }_{4}^{2-}\left(\mathrm{mg} . \mathrm{L}^{-1}\right)$ & 60,79 & 260,4 & 2,330 \\
$\mathrm{Cl}^{-}\left(\mathrm{mg} . \mathrm{L}^{-1}\right)$ & 45,16 & 369,9 & 3,470 \\
Sólidos Totais $\left(\mathrm{mg} . \mathrm{L}^{-1}\right)$ & 13,00 & 337,3 & 78,00 \\
Sólidos Suspensos $\left(\mathrm{mg} . \mathrm{L}^{-1}\right)$ & 12,00 & 122,5 & 50,00 \\
$\mathrm{CO}_{2}$ livre $\left(\mathrm{mg} . \mathrm{L}^{-1}\right)$ & Não obtido & Não obtido & 3,18 \\
$\mathrm{COD}$, (mg. $\left.\mathrm{L}^{-1}\right)$ & 1,01 & 24,1 & 8,84 \\
\hline
\end{tabular}

As determinações de COD foram realizadas utilizando-se um sistema de análise por injeção em fluxo, a partir de uma cela de condutividade, onde se tem o registro da formação do carbono orgânico total (Fadini, 1995). A concentração de COD foi determinada a partir de uma curva analítica, como mostra o gráfico da Figura 1.

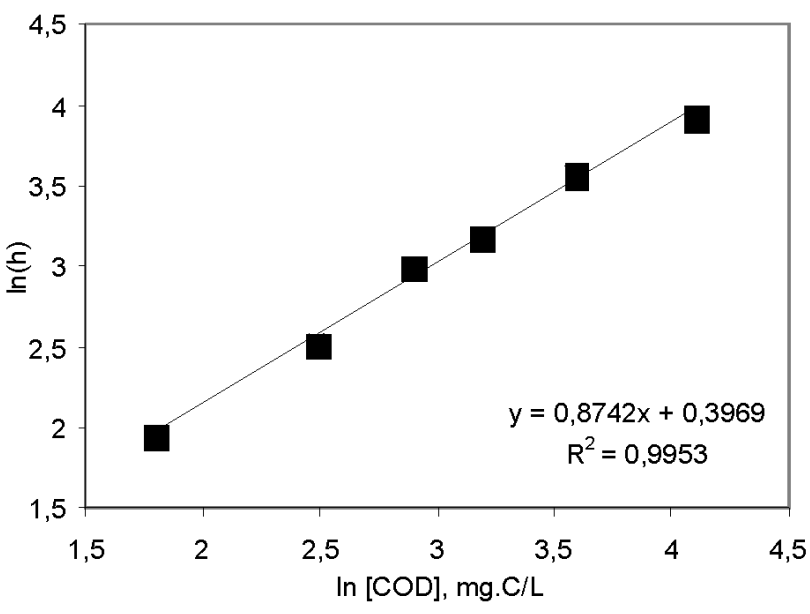

Figura 1 - Curva analítica para a determinação do Carbono Orgânico Dissolvido - COD no sistema de análise por injeção em fluxo, onde h é a altura do pico registrado.

Curvas analíticas dos íons $\mathrm{Cu}$ (II), $\mathrm{Cd}(\mathrm{II})$ e $\mathrm{Pb}(\mathrm{II})$ : as titulaçôes foram realizadas a partir da adição de concentrações conhecidas destes metais, em solução com força iônica variada $\left(y=0,1,0,01\right.$ e 0,001 mol.L $\mathrm{L}^{-1}$ de $\left.\mathrm{NaNO}_{3}\right)$. Utilizou-se três forças iônicas diferentes com variação de grandeza da ordem de 100, para incluir faixa de força iônica das águas típicas de corpos aquáticos. Todas as soluções continham tampão PIPES, de concentração $7,5 \times 10^{-5} \mathrm{~mol} \mathrm{~L}^{-1}$, de forma que o $\mathrm{pH}$ se mantivesse fixo em 6,0. Os cálculos da inclinação da reta foram obtidos a partir da equação da reta: $\mathrm{Y}=\mathrm{bX}+\mathrm{a}$, onde "a" e "b" são, respectivamente, os coeficientes linear e angular da reta. Os gráficos foram construídos com os pontos $\left(\log [\mathrm{M}]_{\mathrm{t}}, \mathrm{E}(\mathrm{mV})\right)$, onde $[\mathrm{M}]_{\mathfrak{r}}$, expressa a concentração do metal total, e "E" o potencial $(\mathrm{mV})$ gerado pelo metal livre. Em todos os casos os valores experimentais obtidos para a inclinação ficou bastante próximo de $29,50 \mathrm{mV}$, o qual é o previsto pela equação de Nernst: $\mathrm{E}=\mathrm{E}^{\mathrm{o}}+(0,0592 / 2) \log \left[\mathrm{M}^{\mathrm{n}+}\right]$, para íons divalentes. As soluçōes titulantes $\left(1,0 \times 10^{-3} \mathrm{~mol} \mathrm{~L}^{-1}\right)$ foram preparadas a partir das suas respectivas soluções padrão. Cada meio foi titulado com os três íons metálicos ( $\mathrm{Cu}(\mathrm{II})$, $\mathrm{Cd}(\mathrm{II})$ e $\mathrm{Pb}(\mathrm{II})$ ), separadamente, anotando-se o potencial em função do volume da solução do íon adicionado. A partir dos resultados, foram construídas curvas analíticas relativas a cada força iônica e para cada íon metálico. Os experimentos foram realizados em triplicata.

Interação dos Ácidos Húmicos com os metais: diferentes concentrações de ácidos húmicos comerciais $(5,0 ; 10,0 ; 20,0$ $\mathrm{mg} \mathrm{L}^{-1}$ ) foram tituladas, com soluções dos íons cobre(II), cádmio(II) e chumbo(II), separadamente, e em três diferentes concentrações de eletrólito $(0,1 ; 0,01$ e $0,001 \mathrm{~mol}$ 
$\mathrm{L}$ de $\mathrm{NaNO}_{3}$ ), de forma a obter as melhores condições para determinação da constante de estabilidade condicional do complexo formado entre a matéria orgânica e o metal. Em cada experimento, utilizou-se o eletrodo sensível ao próprio metal. As titulaçôes foram feitas inicialmente, sem ajuste do $\mathrm{pH}$ e depois ajustando-se o $\mathrm{pH}$ da solução titulada para 6,0 com tampão PIPES $7,5 \times 10^{-5} \mathrm{~mol} \mathrm{~L}^{-1}$. Inicialmente o sistema foi deixado sob agitação por uma hora, para estabilização do potencial inicial. Em seguida prosseguia-se a titulação, com tempo entre cada adição de titulante entre 3 e 10 minutos.

Titulação das Amostras Coletadas nos Rios: Amostras filtradas (sob vácuo, com membranas de acetato de celulose de $0,45 \mu \mathrm{m}$ de porosidade e sistema de filtração de polietileno) foram tituladas adicionando-se com uma microbureta pequenos volumes $(0,01 \mathrm{~mL})$ de solução padrão de cobre, cádmio ou chumbo a alíquota das amostras de $100 \mathrm{~mL}$ já tamponadas (tampão PIPES 7,50 x 10-3 $\mathrm{mol} \mathrm{L}^{-1}$ ) e com força iônica ajustada $\left(\mathrm{NaNO}_{3} 0,01 \mathrm{~mol} \cdot \mathrm{L}^{-1}\right)$. O pH foi ajustado para 6,0 com adição de pequenos volumes de solução $\mathrm{HNO}_{3}$ ou $\mathrm{NaOH} 0,01 \mathrm{~mol} \mathrm{~L}^{-1}$. O tempo decorrido para adição de cada volume do íon metálico variava entre 3 a 10 minutos.

\section{RESULTADOS E DISCUSSÃO}

\section{CURVAS DE TITULAÇÃO DO ELETRÓLITO COM OS ÍONS METÁLICOS}

A Figura 2 (A, B, C) mostra os resultados da titulação com íons $\mathrm{Cu}(\mathrm{II}), \mathrm{Cd}(\mathrm{II})$ e $\mathrm{Pb}(\mathrm{II})$, respectivamente, em diferentes concentrações de eletrólito, sob condições tamponadas $(\mathrm{pH}=$ 6,0 ) e ausência de matéria orgânica. As inclinaçōes obtidas são próximas de $28 \mathrm{mV}$, o que está de acordo com o previsto pela equação de Nernst, para processos envolvendo dois elétrons.

Os gráficos demonstram a dependência do potencial medido com a força iônica do meio, e revela um crescimento linear do mesmo em função da concentração do metal livre. Para os dados em triplicata, o que apresentou melhor definição e reprodutibilidade foram os obtidos em força iônica igual a 0,01 . Entretanto, os experimentos de adição dos metais em uma suspensão com os ácidos húmicos comerciais $(\mathrm{AH})$ foram conduzidos nas três situaçôes de força iônica. Nestes experimentos, os gráficos apresentados na Figura 2 serviram de curvas guia.

A Figura 3 mostra a variação da concentração total do $\mathrm{Cu}$ (II) em função do potencial $(\mathrm{mV})$ das amostras de $\mathrm{AH}$ em três diferentes concentraçōes $\left(5,10\right.$ e $\left.20 \mathrm{mg} \mathrm{L}^{-1}\right)$. Experimentos semelhantes foram conduzidos também com os íons de metais $\mathrm{Cd}(\mathrm{II})$ e $\mathrm{Pb}$ (II). Desnecessário ilustrar os gráficos, mas os resultados estão resumidos na Tabela 2.

Observa-se que em relação aos potenciais obtidos para a construção da curva guia, situação em que o metal estava livre em solução, há uma diminuição devido a complexação
(A)



(B)

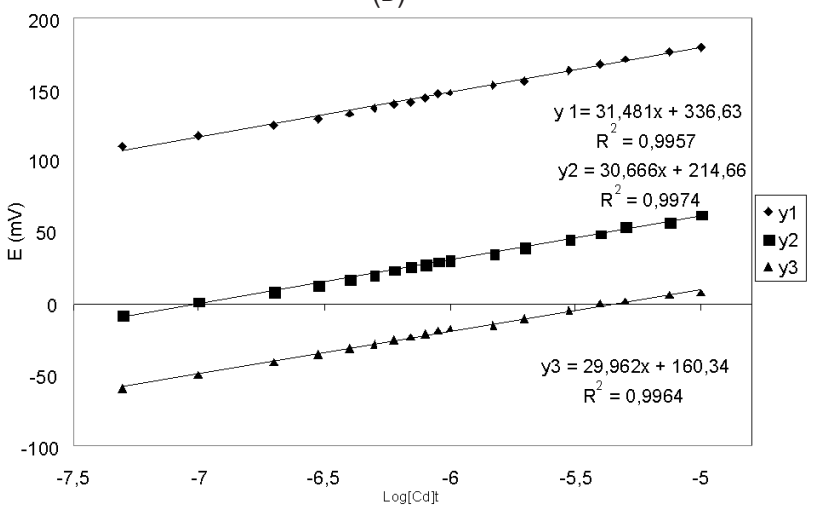

(C)

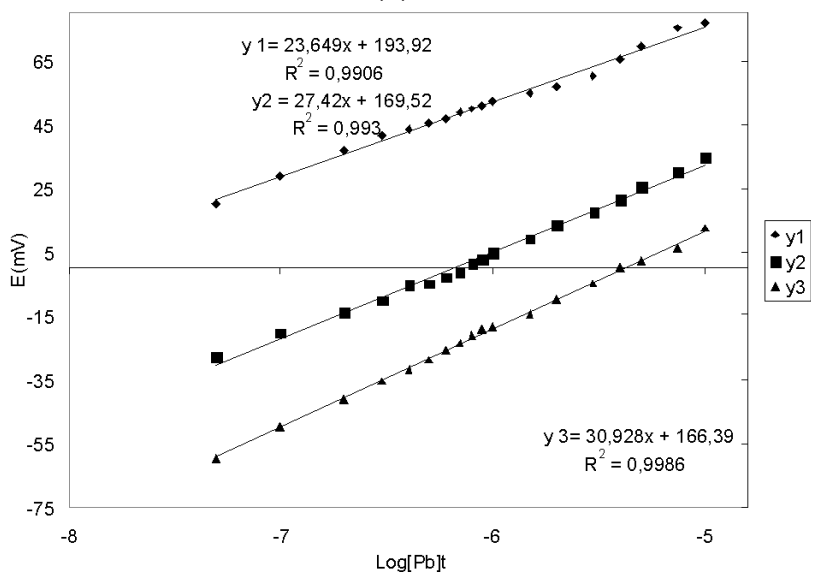

Figura 2 - Curvas de titulação dos íons de metais: A) $\mathrm{Cu}(\mathrm{II})$; B) $\mathrm{Cd}(\mathrm{II})$; C) $\mathrm{Pb}(\mathrm{II})$, em em $\mathrm{pH}=6,0$ e em diferentes concentrações de eletrólito $(y 1=$ 0,$001 ; \mathrm{y} 2=0,01$ e y3 $\left.=0,1 \mathrm{~mol}^{-L^{-1}}\right)$.

do íon de metal, no caso $\mathrm{Cu}(\mathrm{II})$, com a matéria orgânica (CuMOD). Esses experimentos permitiram as determinaçôes das quantidades de metal livre e complexado à MOD, para as diferentes condições de força iônica e concentração de ácidos húmicos. Em seguida, os dados foram tratados pelo método de van den Berg e Kramer (Ellwood e van den Berg, 2000; van den Berg e Kramer, 1979), para a determinação da constante 


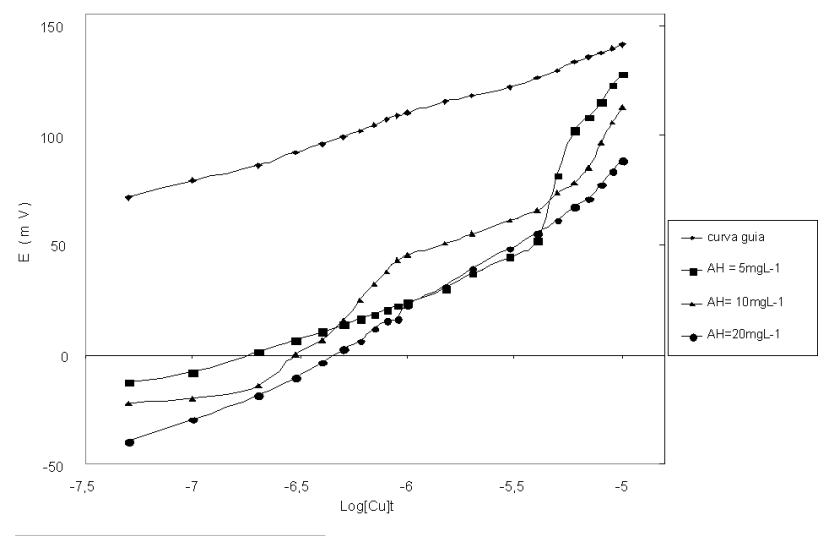

Figura 3 - Efeito da adição do íon Cu(II) em amostras de ácidos húmicos. Condições: $\mathrm{pH}=6,0 ; \mathrm{y}=0,01 \mathrm{~mol}^{-\mathrm{L}^{-1}} \mathrm{de} \mathrm{NaNO}_{3}$.

de formação condicional ( $\left.\mathrm{K}^{\prime}\right)$ e da capacidade de complexação (L) para um sítio de ligação da matéria orgânica dissolvida.

Os gráficos da Figura 4 demonstram a utilização do referido modelo na titulação do íon cobre, em força iônica de $0,01 \mathrm{~mol} \mathrm{~L}^{-1}$ de $\mathrm{NaNO}_{3}$, e para diferentes concentraçôes de ácidos húmicos.

Os resultados obtidos para todos os metais nas diferentes concentrações de AH e força iônica estão sumarizados na Tabela 2.

Para um mesmo metal, os valores determinados para a capacidade de complexação $(\mathrm{Lt})$, não apresentaram uma variação significativa em função da concentração dos ácidos húmicos, especialmente para os cátions $\mathrm{Cd}(\mathrm{II})$ e $\mathrm{Pb}(\mathrm{II})$. Os íons $\mathrm{Cu}(\mathrm{II})$, apresentaram maiores valores de Lt, e uma maior variação destes valores em função da concentração dos ácidos húmicos. Provavelmente por apresentar menor raio iônico que os demais aqui estudados, o íon $\mathrm{Cu}(\mathrm{II})$ interagiu mais e em maior intensidade (K') com a matéria orgânica presente nos ácidos húmicos.

De acordo com os dados obtidos, os valores de Lt apresentaram-se todos na ordem de $10^{-5}$ a $10^{-6}$ mol.. $\mathrm{L}^{-1}$, em conformidade com resultados da literatura (Ellwood e van den Berg, 2000; van den Berg e Kramer, 1979). Isto sugere que o modelo aqui utilizado pode ser aplicado para um sítio de ligação. Quanto à constante de formação condicional, observou-se que esta se manteve próxima de $10^{7}$ a $10^{8}\left(\log K^{\prime}\right.$ variou de 7 a 8), o que demonstra afinidade entre os metais e a matéria orgânica e que os complexos formados são estáveis.

Para a avaliação da relação entre a capacidade de complexação dos ácidos húmicos em função da sua concentração no meio, foram construídos os gráficos Lt x $\mathrm{C}_{\mathrm{AH}}$, os quais estâo ilustrados na Figura 5. Os resultados indicam, que a capacidade de complexação aumenta com o aumento da concentração de ácido húmico. Entretanto, essa relação não é linear e pode estar relacionada à heterogeneidade
(A)

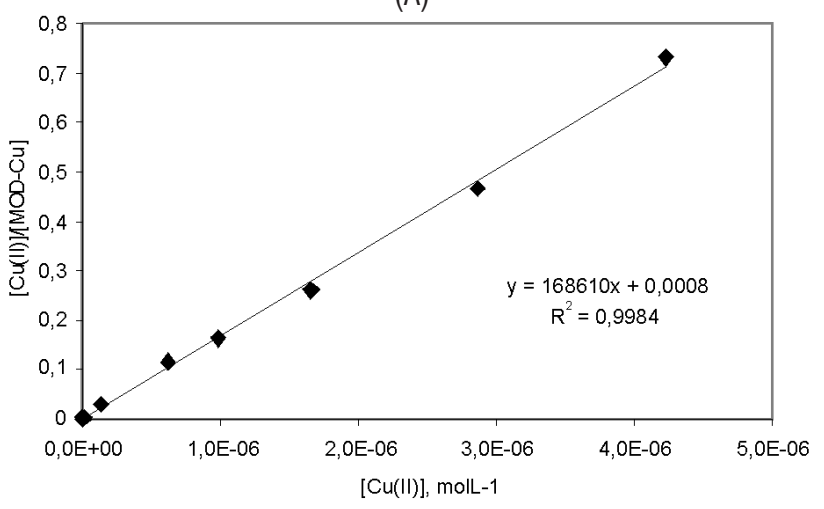

(B)

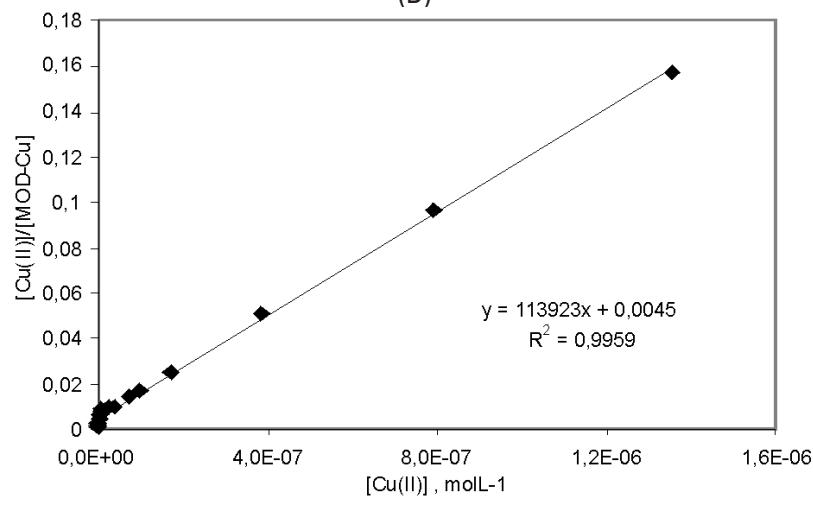

(C)

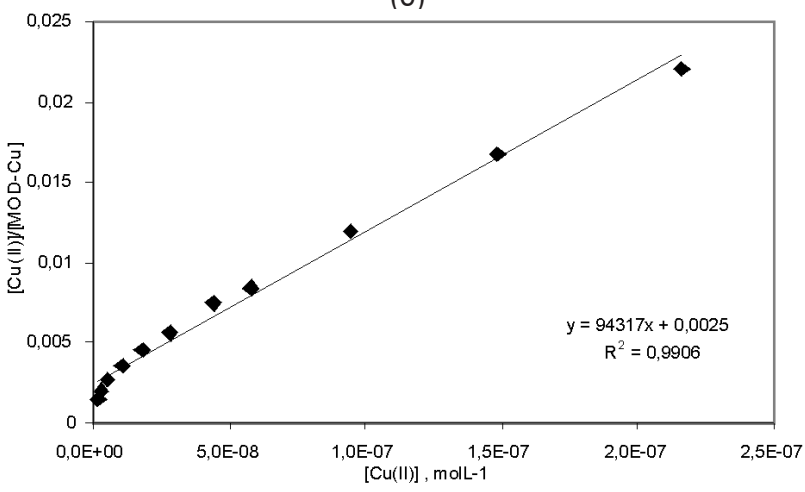

Figura 4 - Modelo de van den Berg e Kramer para titulação potenciométrica da solução de $\mathrm{Cu}$ (II) em ácidos húmicos, pH 6,0 e $/=0,01$ mol.L-1. A) $C_{A H}=$ $5 \mathrm{mg} \cdot \mathrm{L}^{-1}$; B) $C_{\text {AH }}=10 \mathrm{mg} \cdot \mathrm{L}^{-1}$; C) $C_{\text {AH }}=20 \mathrm{mg} \cdot \mathrm{L}^{-1}$.

dos sítios de complexação. Pode-se notar na Figura 4 (B e $\mathrm{C}$, para maiores concentraçôes de ácido húmico) que há um desvio da linearidade no gráfico do método de van den Berg \& Kramer (1979) para pequenos valores de M/ML, ou seja, para maiores valores de ML. Isso indica que após a ocupação dos sítios preferenciais (maior porção linear da curva), o metal passa a complexar com outro tipo de sítio. Borges et al. (2007) e Christensen \& Christensen (2000) abordam a avaliação da complexação de metais em corpos aquáticos com 
Tabela 2 - Valores dos Logarítmos das Constantes de Estabilidade condicional (K') para os complexos (M-MOD; M(II) = Cu, Cd e Pb), Capacidades de Complexação (Lt ) em função da concentração de ácidos húmicos e da força iônica.

\begin{tabular}{|c|c|c|c|c|c|}
\hline \multirow{2}{*}{$y^{1}$} & \multirow{2}{*}{ Parâmetros } & \multirow{2}{*}{$\mathrm{C}_{\mathrm{AH}}\left(\mathrm{mg} \cdot \mathrm{L}^{-1}\right)$} & \multicolumn{3}{|c|}{ Íons de metais M(II) } \\
\hline & & & $\mathrm{Cu}(\mathrm{II})$ & $\mathrm{Cd}(\mathrm{II})$ & $\mathrm{Pb}(\mathrm{II})$ \\
\hline \multirow{6}{*}{ 형 } & \multirow{3}{*}{ Lt $\left(\mu \mathrm{mol} . \mathrm{L}^{-1}\right)$} & 5,0 & $5,93 \pm 0,72$ & $1,00 \pm 0,16$ & $1,19 \pm 0,09$ \\
\hline & & 10,0 & $7,46 \pm 0,63$ & $1,10 \pm 0,12$ & $1,93 \pm 0,10$ \\
\hline & & 20,0 & $9,73 \pm 0,70$ & $1,20 \pm 0,12$ & $3,86 \pm 0,10$ \\
\hline & \multirow{3}{*}{ Log K' } & 5,0 & 8,32 & 7,57 & 6,85 \\
\hline & & 10,0 & 8,47 & 7,35 & 6,92 \\
\hline & & 20,0 & 7,86 & 7,68 & 6,34 \\
\hline \multirow{6}{*}{ 웅 } & \multirow{3}{*}{$\mathrm{Lt}\left(\mu \mathrm{mol} \cdot \mathrm{L}^{-1}\right)$} & 5,0 & $3,13 \pm 0,30$ & $0,33 \pm 0,02$ & $0,24 \pm 0,01$ \\
\hline & & 10,0 & $6,52 \pm 0,50$ & $0,66 \pm 0,01$ & $0,50 \pm 0,02$ \\
\hline & & 20,0 & $9,50 \pm 0,55$ & $1,17 \pm 0,08$ & $1,00 \pm 0,01$ \\
\hline & \multirow{3}{*}{ Log K' } & 5,0 & 6,67 & 7,26 & 6,87 \\
\hline & & 10,0 & 7,97 & 7,07 & 7,18 \\
\hline & & 20,0 & 6,72 & 7,10 & 7,34 \\
\hline \multirow{6}{*}{$\overline{0}$} & \multirow{3}{*}{ Lt $\left(\mu \mathrm{mol} \cdot \mathrm{L}^{-1}\right)$} & 5,0 & $7,46 \pm 0,62$ & $0,47 \pm 0,02$ & $2,12 \pm 0,12$ \\
\hline & & 10,0 & $9,36 \pm 0,62$ & $0,50 \pm 0,02$ & $3,21 \pm 0,10$ \\
\hline & & 20,0 & $10,2 \pm 0,52$ & $0,52 \pm 0,02$ & $4,56 \pm 0,10$ \\
\hline & \multirow{3}{*}{ Log K' } & 5,0 & 7,37 & 6,83 & 6,73 \\
\hline & & 10,0 & 7,77 & 7,13 & 6,98 \\
\hline & & 20,0 & 8,38 & 6,89 & 6,94 \\
\hline
\end{tabular}

a matéria orgânica dissolvida baseada em 2 sítios de ligação. Os últimos autores demonstram que também que não há uma relação linear entre a quantidade de metal complexado e a concentração de MOD. Ao aumentar a concentração de ácido húmico, a capacidade de complexação passa a não responder linearmente devido aos metais apresentarem afinidades diferentes com relação ao sítio preferencial de ligação, influenciando a inclinação da reta no gráfico de Lt versus concentração de ácido húmico. No tratamento dos dados considerou-se 1 sítio de ligação, por este ser preferencial e de maior relevância para o controle de metais em corpos d'água, tanto em termos de estabilidade como de capacidade de complexação.

Os teores de carbono orgânico dissolvido (COD) presentes nos ácidos húmicos e para as concentrações de 5,0; 10,0 e 20,0 $\mathrm{mg} \mathrm{L}^{-1}$, foram determinados e são respectivamente iguais a: 0,$82 ; 1,41$ e $2,53 \mathrm{mgC} \mathrm{L}^{-1}$. Estes valores foram relacionados com a capacidade de complexação para os diversos casos. De acordo com os resultados obtidos, houve apenas um ligeiro acréscimo de capacidade de complexação dos ácidos húmicos em função da duplicação da concentração dos ácidos húmicos, pelo mesmo motivo explanado na relação entre Lt e a concentração de ácido húmico. Os resultados para força iônica de 0,01 mol.L-1 estão apresentados na Figura 6.

CURVAS DE TITULAÇÃO DAS AMOSTRAS REAIS COM OS ÍONS
(A)

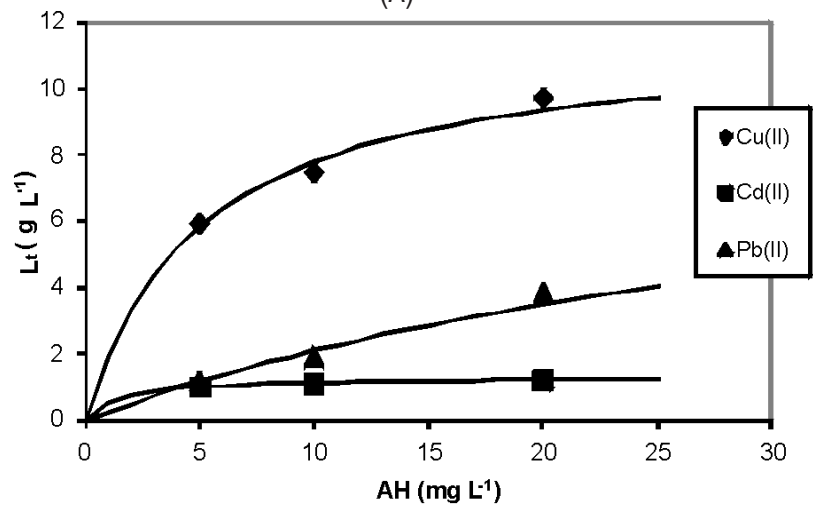

(B)

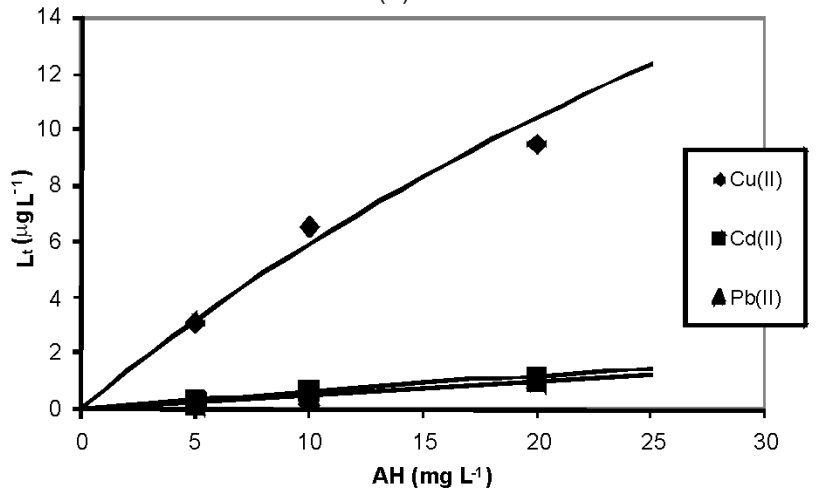


(C)

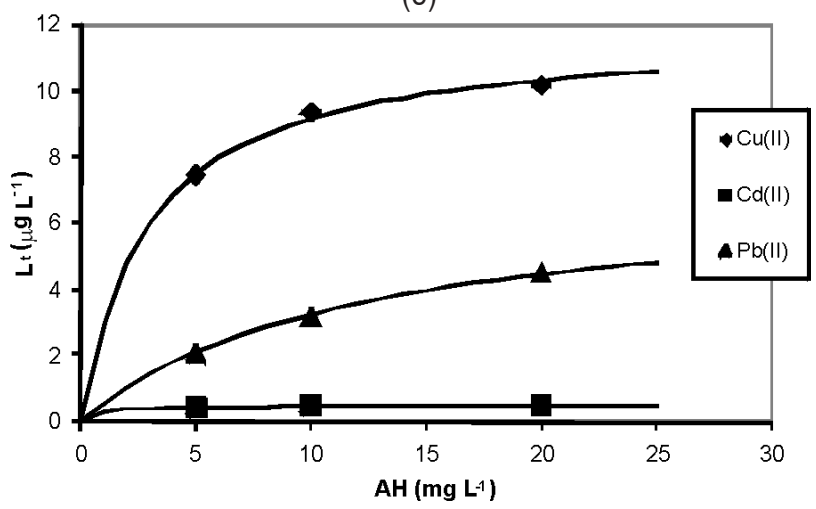

Figura 5 - Relação entre a concentração de ácidos húmicos $\left(\mathrm{C}_{\mathrm{AH}}\right)$ e a capacidade de complexação(Lt) dos íons metálicos $\mathrm{Cu}(\mathrm{II}), \mathrm{Cd}(\mathrm{II})$ e $\mathrm{Pb}(\mathrm{II})$. pH $=6,0$ e força iônica: A) 0,001 mol.L-1' B) 0,01 mol.L-1; C) 0,1 mol.L-1.

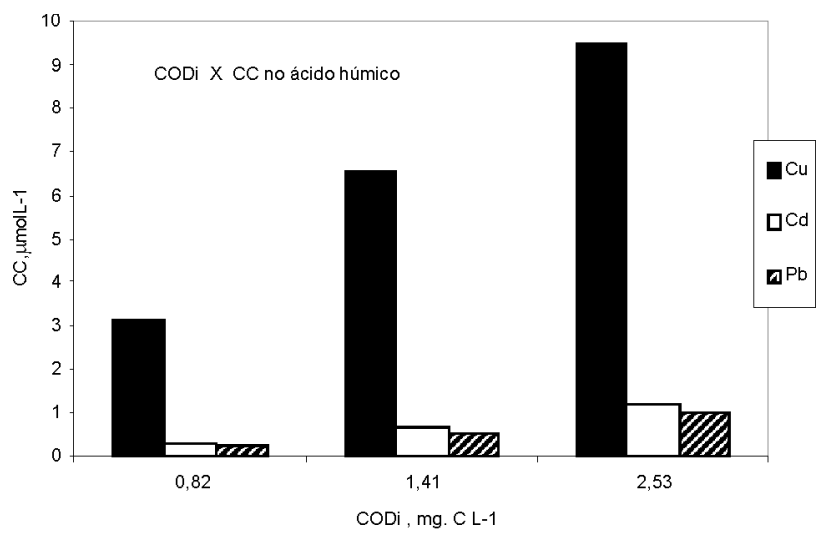

Figura 6 - Relação entre a capacidade de complexação e a concentração de carbono orgânico dissolvido (COD) nos ácidos húmicos para força iônica de $0,01 \mathrm{~mol} . \mathrm{L}^{-1}$

\section{METÁLICOS}

Após a verificação do modelo de van den Berg e Kramer aplicado aos íons metálicos seletivos com ácido húmico, empregou-se este estudo nas interações entre a matéria orgânica dissolvida em águas de rios maranhenses e os íons $\mathrm{Cu}(\mathrm{II}), \mathrm{Cd}(\mathrm{II})$ e $\mathrm{Pb}(\mathrm{II})$. As amostras reais foram coletadas nos rios Pericumã, Bacanga e Itapecuru. Estas amostras foram submetidas ao mesmo tratamento que os eletrólitos apresentados anteriormente.

A Figura 7 mostra os resultados da titulação com o eletrodo sensível a cobre, o que denota a formação de um complexo, pois há um afastamento da curva guia, o que significa que uma fração do cobre foi complexada ou adsorvida. De acordo com esta Figura, a amostra do Rio Pericumã tem uma variação de potencial inferior em relação as amostras dos demais rios. Esta variação, que está relacionada com a quantidade de complexo formado, é justificada pela quantidade de matéria orgânica dissolvida. Conforme descrito na Tabela 1, a quantidade de carbono orgânico dissolvido (COD) é maior para o Rio Bacanga, seguido do Rio Itapecuru, e finalmente do Rio Pericumã. Os valores dos potenciais experimentais mostrados na Figura 7, em relação a curva guia, refletem, portanto, esta tendência.

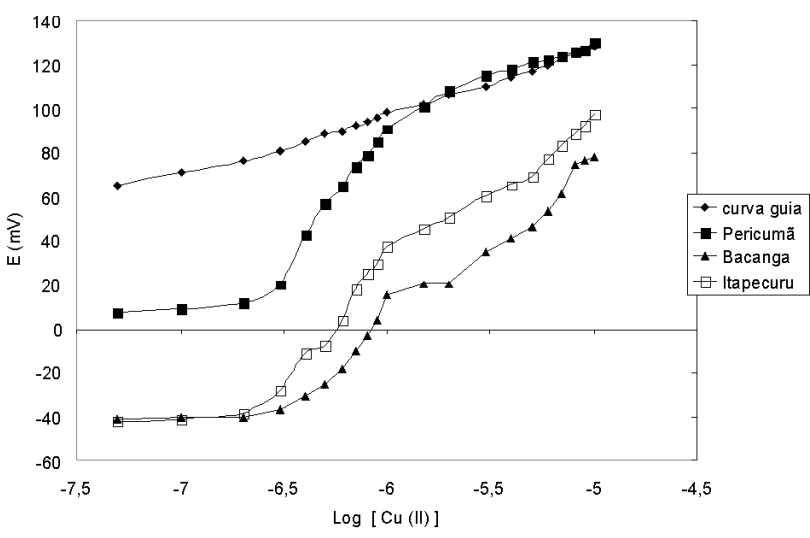

Figura 7 - Variação das concentrações de cobre nas amostras de águas superficiais: Rio Pericumã, Rio Bacanga e Rio Itapecuru $\mathrm{pH}=6,0, y=$ 0,01 mol.L-1 de $\mathrm{NaNO}_{3}$

Os gráficos da Figura 8 são referentes ao tratamento dos resultados da Figura 7, os quais, a partir destes, foram determinadas capacidades de complexação e constantes de estabilidades condicional das amostras. Os resultados para todos os íons estão apresentados na Tabela 3.

Tabela 3 - Valores dos Logaritmos das Constantes de Estabilidade condicional (K') para os complexos (M-MOD; $\mathrm{M}(\mathrm{II})=\mathrm{Cu}, \mathrm{Cd}$ e Pb), Capacidades de Complexação (Lt) e quantidades de Carbono orgânico dissolvido (COD) para as amostras dos Rios.

\begin{tabular}{|c|c|c|c|c|c|}
\hline \multirow{2}{*}{ Rio } & \multirow{2}{*}{$\begin{array}{c}\text { COD } \\
\left(\mathrm{mg} . \mathrm{L}^{-1}\right)\end{array}$} & \multirow{2}{*}{ Parâmetros } & \multicolumn{3}{|c|}{ ĺons de metais M(II) } \\
\hline & & & $\mathrm{Cu}(\mathrm{II})$ & $\mathrm{Cd}(\mathrm{II})$ & $\mathrm{Pb}(\mathrm{II})$ \\
\hline \multirow{2}{*}{ 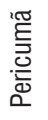 } & \multirow{2}{*}{1,01} & $\mathrm{Lt}\left(\mu \mathrm{mol} \cdot \mathrm{L}^{-1}\right)$ & 0,35 & 0,10 & 0,82 \\
\hline & & Log K' & 7,00 & 7,40 & 6,99 \\
\hline \multirow{2}{*}{ 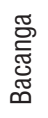 } & \multirow{2}{*}{24,1} & $\mathrm{Lt}\left(\mu \mathrm{mol} \cdot \mathrm{L}^{-1}\right)$ & 1,03 & 1,00 & 2,69 \\
\hline & & Log K' & 7,20 & 6,98 & 6,71 \\
\hline \multirow{2}{*}{ 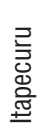 } & \multirow{2}{*}{8,84} & $\mathrm{Lt}\left(\mu \mathrm{mol} \cdot \mathrm{L}^{-1}\right)$ & 0,50 & 0,33 & 2,67 \\
\hline & & Log K' & 7,76 & 7,04 & 6,69 \\
\hline
\end{tabular}

De acordo com os valores da Tabela 3, segundo o modelo de van den Berg e Kramer, o rio Pericumã apresenta-se com menor capacidade de complexação e quantidade de carbono orgânico dissolvido. Isto se confirma na análise com todos os íons metálicos estudados. O rio Bacanga possui uma concentração mais elevada de carbono orgânico dissolvido, $24,08 \mathrm{mg} \mathrm{L}^{-1}$, e capacidade de complexação maior quando 
(A)

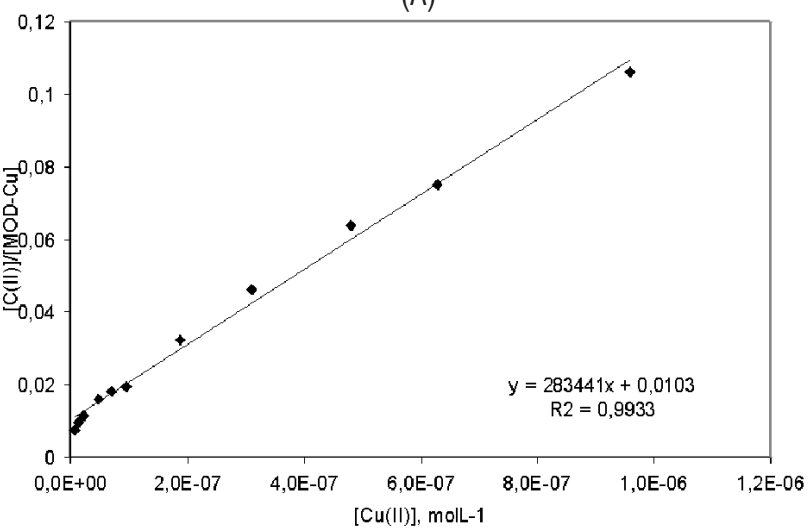

(B)

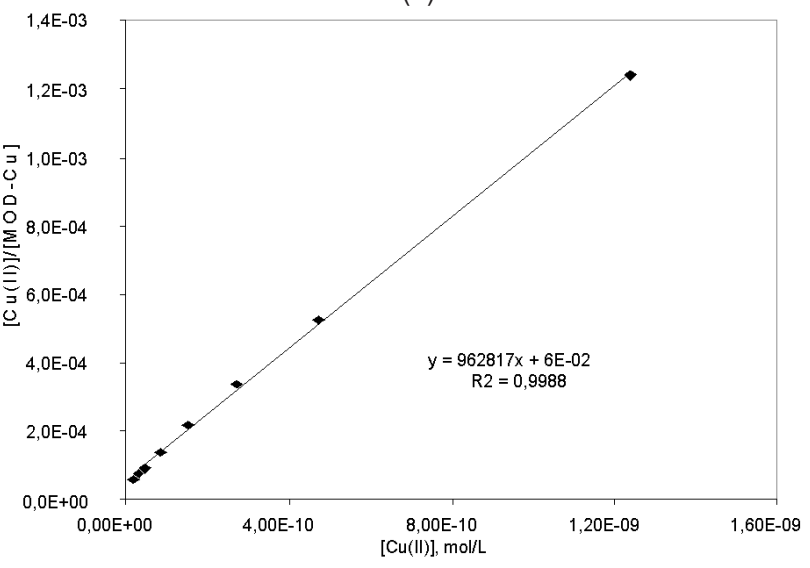

(C)

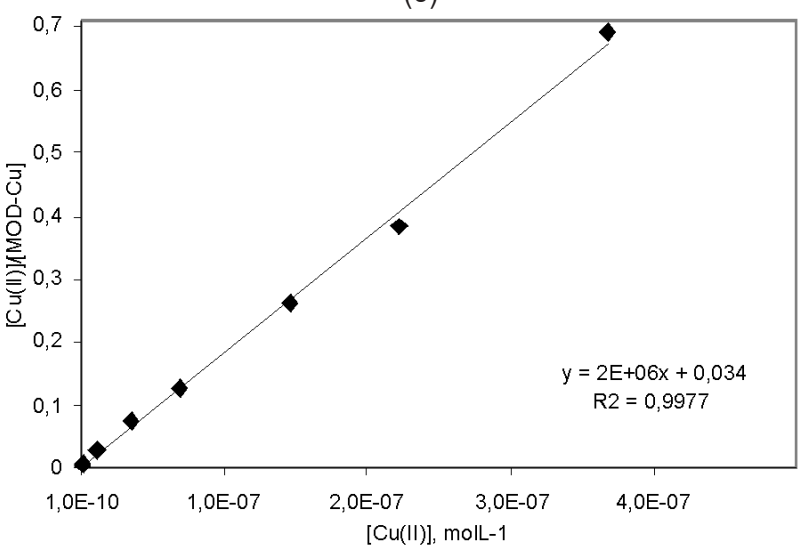

Figura 8 - Aplicação do Modelo de van den Berg e Kramer na titulação potenciométrica da solução de $\mathrm{Cu}(\mathrm{II})$, em amostra do Rio: A) Pericumã; B) Bacanga; C) Itapecuru.

comparado aos outros rios. Provavelmente, isto se relaciona com o fato deste rio possuir, próximo ao seu leito grande quantidade de residências o que resulta em sua poluição, com dejetos, animais mortos, pedaços de arvores, folhas etc, e com decomposição destes, ocorre o aumento da concentração da matéria orgânica no meio. Outro fato que pode ser citado é a sua ligação com mar, este é rico em eletrólitos, o que resulta em maior força iônica, em relação aos demais.

A seguir, a Figura 9 relaciona a capacidade de complexação encontrado para cada amostra com as quantidades respectivas de carbono orgânico dissolvido.

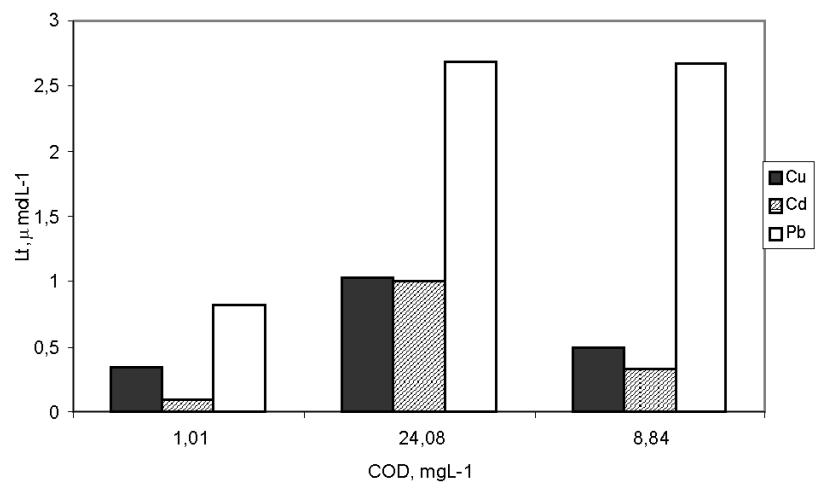

Figura 9 - Relação entre $L_{t}$ e COD nas amostras de águas superficiais (rios Pericumã, Bacanga e Itapecuru).

Comparando os resultados apresentados na Figura 9 (amostra de águas superficiais) com os apresentados na Figura 6 (simulaçôes com ácidos húmicos), nota-se que a capacidade de complexação dos íons $\mathrm{Cu}(\mathrm{II})$ é menor para as amostras dos rios do que para as amostras de ácidos húmicos. Este fato está associado a um fator importante que diz respeito à maior ocorrência do $\mathrm{Cu}(\mathrm{II})$ em ambientes aquáticos que os íons $\mathrm{Cd}(\mathrm{II})$ e $\mathrm{Pb}(\mathrm{II})$. Em relação ao $\mathrm{Cd}(\mathrm{II})$, pode-se dizer que se apresentou como sendo o íon mais biodisponível em amostras de águas superficiais, pois as "capacidades de complexação" apresentaram valores menores que os Lt dos outros metais. Na Tabela 3, demonstra-se que na comparação de Lt e COD, para o íon cádmio e cobre, verifica-se que existe coerência de resultados, isto é, quanto maior a quantidade de carbono orgânico maior a capacidade de ligação da matéria orgânica dissolvida com os íons $\mathrm{Cd}(\mathrm{II})$ e $\mathrm{Cu}(\mathrm{II})$. Quanto ao $\mathrm{Pb}$ (II), este metal não seguiu a mesma tendência, ou seja, não houve um aumento significativo da Lt com a concentração de COD dos rios; mas, o chumbo apresentou as maiores capacidades de complexação entre os metais estudados. Os resultados sugerem que há presença de ligantes nas amostras naturais com excepcional afinidade pelos íons $\mathrm{Pb}(\mathrm{II})$, tais como $\mathrm{Cl}^{-} \mathrm{e} \mathrm{SO}_{4}^{2-}$ (ver Tabela 1) que também se ligam ao íon metálico, contribuindo para os resultados da capacidade de complexação; isto pode também ser verificado pela diminuição considerável da quantidade de íon livre de chumbo na solução, determinada pelo potencial medido (Ruzic, 1980).

\section{CONCLUSÕES}

Do conjunto de experimentos e estudos aqui realizados, envolvendo a interação $\mathrm{M}-(\mathrm{MOD}, \mathrm{COD})$, onde $\mathrm{M}=$ 
$\mathrm{Cu}(\mathrm{II}), \mathrm{Cd}(\mathrm{II})$ e $\mathrm{Pb}(\mathrm{II})$ e $\mathrm{MOD}, \mathrm{COD}$ provenientes de produtos de substâncias húmicas de amostras naturais (Rios Itapecuru, Pericumã e Bacanga), pode-se fazer as seguintes consideraçōes:

Houve um aumento da capacidade de complexação dos íons estudados com o aumento dos teores de MOD e COD. A relação não linear indica a presença de sítios discretos de complexação; entretanto, a presença de metais na forma livre em corpos aquáticos é controlada pelo sítio preferencial de complexação.

O método de van den Berg e Kramer, para um sítio de coordenação, mostrou-se adequado para descrever a interação dos íons investigados com ácidos húmicos. Os valores dos parâmetros estimados por este modelo (K’ e Lt) estão de acordo com os reportados na literatura.

Devido influência da presença de outros íons metálicos, por competição ou alterações das cargas no ambiente, os valores de Lt nas amostras se apresentaram diferentes das simulaçôes feitas com ácidos húmicos, levando-se também em conta a natureza de cada metal.

O íon de cádmio se apresentou o mais biodisponível nas amostras dos rios Itapecuru, Bacanga e Pericumã.

A capacidade de complexação da matéria orgânica dissolvida com íon metálico chumbo, apresentou-se muito alta em relação a simulação com $\mathrm{AH}$, isto devido a presença de outros ligantes, que complexa com este metal.

Em ambientes naturais, devido à complexidade da matéria orgânica, há competição entre os diversos ligantes (incluindo outros sítios de complexação) pelos íons metálicos, portanto, a correlação direta $(\mathrm{MOD}, \mathrm{COD})$ versus $\mathrm{Lt}$ merece maiores cuidados.

\section{AGRADECIMENTOS}

Os autores agradecem o apoio financeiro recebido das agências CAPES e CNPq.

\section{BIBLIOGRAFIA CITADA}

Abdel, O.; Nicolas, P.; Gabriel, B.; Philippe, R.; Baghdad, O. 2007. Environmental impacts of heavy metal discharges from a smelter in Deûle-canal sediments (Northern France): Concentration levels and chemical fractionation. Water, air and soil pollution, 180: 83-95.

Allen, H. E.; Hansen, D. J. 1996. The importance of chemical speciation to water quality criteria. Water Environment, 68(1): 42-54.

Arcos, A. S. V. 1998. Determinação de carbono orgânico dissolvido através de um sistema de análise por injeção em fluxo (FIA) e foto-fenton. Dissertação de Mestrado, Universidade Federal do Maranhão, São Luís, Maranhão. 69pp.
Borges, E. C. L.; Mozeto A. A.; Neves, E. F. A.; Borges Neto, W. B.; Bezerra, J. M. 2007. Estudo da capacidade de complexação e sua relação com algumas variáveis ambientais em cinco represas do Rio Tietê/Brasil. Quim. Nova, 30 (7): 1505-1511.

Cances, B.; Ponthieu, M.; Castrec-Rouelle, M.; Aubry E.; Benedetti, M.F. 2003. Metal ions speciation in a soil and its solution: experimental data and model results. Geoderma, 113 (3): 341 355.

Christensen, J. B.; Christensen, T. H. 2000. The effect of $\mathrm{pH}$ on the complexation of $\mathrm{Cd}, \mathrm{Ni}$ and $\mathrm{Zn}$ by dissolved organic carbon from leachate-polluted groundwater. Water Research, 34(15): 3743-3754.

Ellwood, M. J.; van den Berg, C. M. G. 2000. Zinc speciation in the Northeastern Atlantic Ocean. Marine Chemistry, 68 (4): 295-306.

Fadini, P.S. 1995. Determinação de carbono orgânico dissolvido em amostras naturais através de analise por injeção em fluxo. Dissertação de Mestrado, Faculdade de Engenharia Civil/ Universidade Estadual de Campinas. 104pp.

Martiniano, L. C.; Bezerra, C. W. B.; Marques, E. P.; Sousa, A. G.; Fernandes, R. N. Marques, A. L. B. 2008. Novo método espectrofotométrico para determinação de $\mathrm{Hg}(\mathrm{II})$ em amostras de pexies. Ciência e Tecnologia de Alimentos, 28(2): 375-381.

Nederiof, M. M.; Wit, J. C. M de; Riemsdijk, W. A. V.; Koopal, L. K. 1993. Environmental Science \& Technology, 27: 846-856.

Rocha, J.C.; Dene-Sene, J.J.; Burba, Klockow, P. 1998. Journal of the Brazilian Chemistry Society, 9: 79-84.

Ruzic, I. 1980. Theoretical aspects of the direct titration of natural waters and its information yield for trace metal speciation. Analytica Chimica Acta, 140: 99-113.

Santana, G.; Barroncas, P. S. R. 2007. Estudo de íons de metais (Co, $\mathrm{Cu}, \mathrm{Fe}, \mathrm{Cr}, \mathrm{Ni}, \mathrm{Mn}, \mathrm{Pb}$ e $\mathrm{Zn}$ ) na Bacia do Tarumã-Açu Manaus (AM). Acta Amazonica, 37(1): 111-118.

Sánchez-Marín, P.; Lorenzo, J. I.; Blust, R.; Beiras, R. 2007. Humic Acids Increase Dissolved Lead Bioavailability for Marine Invertebrates. Environmental Science Technology, 41(16): 56795684 .

Shazili, N. A.; Yunus, K.; Ahmad, A.; Abdullah, N.; Rashid, M. 2006. Aquatic Ecosystem Health \& Management, 9(2): 137-145.

Stumm, W.; Morgan, J. J. 1996. Aquatic chemistry - chemical equilibria and rates in natural waters. John Wiley \& Sons, New York, USA. 1022pp.

Stumm, W.1992. Chemistry of the solid water interface - processes at the mineral - water and particle-water-interface in natural systems. John Wiley \& Sons, New York, USA. 428pp.

van den Berg, C. M. G.; Kramer, J. R. 1979. Determination of complexing capacities of ligands in natural waters and constants of conditional stability constants of copper complexes by means of manganese dioxide. Analytica Chimica Acta, 106: 113-120.

Voelker, B. M.; Kogut, M. B. 2001. Interpretation of metal speciation data in coastal waters: the effects of humic substances on copper binding as a test case. Marine Chemistry, 74 (4): 303-318. 
ACTA

AMAZONICA

Westall, J. C. 1995. Models for association of metal ions with heterogeneous environmental sorbents. 1. Complexation of $\mathrm{Co}(\mathrm{II})$ by leonardite humic acid as function of $\mathrm{pH}$ and $\mathrm{NaClO}_{4}$ concentration. Environmental Science \& Technology, 29(4): 951-959.

Recebido em 15/05/2008

Aceito em 29/11/2008 